Vietnam Journal of Mechanics, VAST, Vol.37, No. 2 (2015), pp. 123-132

DOI:10.15625/0866-7136/37/2/5659

\title{
EQUIVALENT-INCLUSION APPROACH FOR ESTIMATING THE ELASTIC MODULI OF MATRIX COMPOSITES WITH NON-CIRCULAR INCLUSIONS
}

\author{
Tran Nguyen Quyet ${ }^{1}$, Pham Duc Chinh ${ }^{2}$, Tran Anh Binh ${ }^{3, *}$ \\ ${ }^{1}$ Hanoi University of Industry, Vietnam \\ ${ }^{2}$ Institute of Mechanics, Vietnam Academy of Science and Technology, Hanoi, Vietnam \\ ${ }^{3}$ National University of Civil Engineering, Hanoi, Vietnam \\ *E-mail: anh-binh.tran@nuce.edu.vn \\ Received December 03, 2014
}

\begin{abstract}
A novel approach to predict the effective elastic moduli of matrix composites made from non-circular inclusions embedded in a continuous matrix is proposed. In this approach, those inhomogeneities are substituted by simple equivalent circularinclusions with modified elastic properties obtained from comparing the dilute solution results. Available simple approximations for the equivalent circular-inclusion medium then can be used to estimate the effective elastic moduli of the original composite. Robustness of proposed approach is demonstrated through the numerical examples with elliptic inclusions.

Keywords: Effective elastic moduli, isotropic multicomponent material, matrix composite, elliptic inclusion, circular inclusion.
\end{abstract}

\section{INTRODUCTION}

Practical materials are often strongly heterogeneous and contain high volume fractions of inclusions with sizes spread over several spatial scales. Regarding the intensive use of these materials, predicting and optimizing their effective properties from a microscopic description represents a considerable industrial interest. In view of the microscopic morphological complexity, analytical results are limited, even in the linear case. Upper and lower bounds on the possible values of the effective properties [1-6] are not very useful in the case of high contrast of matrix-inclusion properties. Numerical homogenization techniques [7-12] allow overcoming these issues, but efficient strategies must be developed to avoid important computational costs, especially in the case of complex three-dimensional microstructure. The effective medium approximations developed over years is useful for engineers due to its simplicity. A straightforward choice is the

(C) 2015 Vietnam Academy of Science and Technology 
volume-weighted averages. More advanced approximations are self-consistent, differential, Mori-Tanaka approximations, correlation approximation, ... [13-20], the latter ones often account just for the shapes of the inhomogeneities and the matrix-inclusion interactions. However, many approximations are actually applicable only for the composites with isotropic inclusions of certain simple shapes. To overcome this issue, we propose here a novel approach for macroscopically isotropic composites containing non-circular inclusions, permitting to substitute those inhomogeneities by isotropic circular inclusions with equivalent elastic properties. Available approximations for circular inclusion composites then can be used to estimate the effective elastic moduli of original media. The paper is organized as follows.

Firstly, the proposed approach is presented in section 2. In the next section, the effective elastic moduli of some matrix composites with elliptic inclusions are numerically investigated. Conclusions are made in the last section.

\section{THE EQUIVALENT-INCLUSION APPROACH}

The approach is expected to apply to composites, containing inclusions of any complicated forms, once dilute solutions for them can be constructed analytically or numerically. Specifically, we restrict ourself here to the 2D elliptic-inclusion composites.

A macroscopically isotropic medium associated with isotropic particles of elliptic shape is considered. The effective elastic moduli of a dilute suspension of those randomly oriented elliptic particles having the elastic moduli $K_{I}, \mu_{I}$ and volume fraction $v_{I}$ in a matrix of elastic properties $K_{M}, \mu_{M}$ have the form [21,22]

$$
\begin{gathered}
K^{e f f}=K_{M}+v_{I}\left(K_{I}-K_{M}\right) K_{D}, \\
\mu^{e f f}=\mu_{M}+v_{I}\left(\mu_{I}-\mu_{M}\right) \mu_{D},
\end{gathered}
$$

where $v_{I} \ll 1, K_{D}$ and $\mu_{D}$ are expressed by

$$
\begin{aligned}
& K_{D}=\frac{\alpha_{\mu}\left(P_{1111}-P_{1122}-P_{2211}+P_{2222}\right)+2}{\widehat{P}}, \\
& \mu_{D}=\frac{\alpha_{K}\left(P_{1111}+P_{1122}+P_{2211}+P_{2222}\right)+2}{2 \widehat{P}}+\frac{1}{2\left(2 \alpha_{\mu} P_{1212}+1\right)}, \\
& \widehat{P}=2 \alpha_{K} \alpha_{\mu}\left(P_{1111} P_{2222}-P_{2211} P_{1122}\right)+\left(\alpha_{K}+\alpha_{\mu}\right)\left(P_{1111}+P_{2222}\right)+ \\
& +\left(\alpha_{K}-\alpha_{\mu}\right)\left(P_{1122}+P_{2211}\right)+2 \\
& \alpha_{K}=\frac{K_{I}}{K_{M}}-1, \quad \alpha_{\mu}=\frac{\mu_{I}}{\mu_{M}}-1,
\end{aligned}
$$

$\mathbf{P}$ is Eshelby tensor defined in [21]

$$
P_{1111}=\frac{K_{M}}{K_{M}+\mu_{M}}\left(\frac{a_{2}^{2}+2 a_{1} a_{2}}{\left(a_{1}+a_{2}\right)^{2}}+\frac{\mu_{M}}{K_{M}} \frac{a_{2}}{a_{1}+a_{2}}\right),
$$




$$
\begin{aligned}
& P_{2222}=\frac{K_{M}}{K_{M}+\mu_{M}}\left(\frac{a_{1}^{2}+2 a_{1} a_{2}}{\left(a_{1}+a_{2}\right)^{2}}+\frac{\mu_{M}}{K_{M}} \frac{a_{1}}{a_{1}+a_{2}}\right), \\
& P_{1122}=\frac{K_{M}}{K_{M}+\mu_{M}}\left(\frac{a_{2}^{2}}{\left(a_{1}+a_{2}\right)^{2}}-\frac{\mu_{M}}{K_{M}} \frac{a_{2}}{a_{1}+a_{2}}\right), \\
& P_{2211}=\frac{K_{M}}{K_{M}+\mu_{M}}\left(\frac{a_{1}^{2}}{\left(a_{1}+a_{2}\right)^{2}}-\frac{\mu_{M}}{K_{M}} \frac{a_{1}}{a_{1}+a_{2}}\right), \\
& P_{1212}=\frac{K_{M}}{K_{M}+\mu_{M}}\left(\frac{a_{1}^{2}+a_{2}^{2}}{2\left(a_{1}+a_{2}\right)^{2}}+\frac{\mu_{M}}{2 K_{M}}\right),
\end{aligned}
$$

where $a_{1}, a_{2}$ are the principal sizes of the elliptic inclusion.

On the other side, the dilute suspension results for isotropic circular particles having moduli $\bar{K}_{I}, \bar{\mu}_{I}$ and volume fraction $v_{I}$ in the matrix of the same elastic properties $K_{M}$, $\mu_{M}$ is expressed by [23]

$$
\begin{aligned}
K^{e f f} & =K_{M}+v_{I}\left(\bar{K}_{I}-K_{M}\right) D_{K}\left(\bar{K}_{I}, \bar{\mu}_{I}, K_{M}, \mu_{M}\right), \\
\mu^{e f f} & =\mu_{M}+v_{I}\left(\bar{\mu}_{I}-\mu_{M}\right) D_{M}\left(\bar{K}_{I}, \bar{\mu}_{I}, K_{M}, \mu_{M}\right),
\end{aligned}
$$

where $D_{K}$ and $D_{M}$ are defined as

$$
\begin{aligned}
D_{K}\left(\bar{K}_{I}, \bar{\mu}_{I}, K_{M}, \mu_{M}\right) & =\frac{K_{M}+\mu_{M}}{\bar{K}_{I}+\mu_{M}}, \\
D_{M}\left(\bar{K}_{I}, \bar{\mu}_{I}, K_{M}, \mu_{M}\right) & =\frac{\mu_{M}+\mu_{*}\left(K_{M}, \mu_{M}\right)}{\bar{\mu}_{I}+\mu_{*}\left(K_{M}, \mu_{M}\right)}, \\
\mu_{*}\left(K_{0}, \mu_{0}\right) & =\frac{\mu_{0} K_{0}}{K_{0}+2 \mu_{0}} .
\end{aligned}
$$

Equalizing (1)-(2) and (11)-(12), one finds

$$
\begin{aligned}
& \bar{K}_{I}=\frac{K_{M}^{2}+\mu_{M} K_{M}+2\left(K_{I}-K_{M}\right) K_{D} \mu_{M}}{\mu_{M}+K_{M}-2\left(K_{I}-K_{M}\right) K_{D}}, \\
& \bar{\mu}_{I}=\frac{\left(\mu_{I}-\mu_{M}\right) \mu_{D} \mu_{M} K_{M}+\mu_{M}\left(\mu_{M} K_{M}+\mu_{M}^{2}\right)}{\mu_{M} K_{M}+\mu_{M}^{2}-\left(\mu_{I}-\mu_{M}\right) \mu_{D}\left(K_{M}+2 \mu_{M}\right)},
\end{aligned}
$$

which are referred to as the moduli of the equivalent circular inclusions for the original elliptic inclusions of elastic moduli $K_{I}$ and $\mu_{I}$. Using (16)-(17), all the elliptic inclusions can be replaced by circular inclusions of the same volumes, but with modified properties. We call it the equivalent-inclusion approach. The main objective of the approach is to substitute the original composite involving elliptic inclusions by an equivalent medium containing circular inclusions, for which many convenient effective medium approximations are available. 


\section{NUMERICAL EXAMPLES FOR EFFECTIVE ELASTIC MODULI}

In this section, we use the Finite Element Method (FEM) to estimate the effective elastic moduli of the elastically-isotropic composites containing elliptic inclusions and those of equivalent composites with circular inclusions. Let us consider an inhomogeneous periodic rectangular domain $\Omega$ with boundary $\partial \Omega$. The strong form associated with the cell is given by

$$
\begin{gathered}
\nabla \cdot \sigma+\mathbf{b}=0 \text { in } \Omega, \\
\sigma=\mathbb{C}: \varepsilon,
\end{gathered}
$$

where $\varepsilon=\left(\nabla \mathbf{u}+\nabla \mathbf{u}^{T}\right) / 2$, and $\mathbb{C}$ is the fourth-order tensor of elastic moduli. The displacement field on the cell boundary is constrained in terms of the macroscopic strain $\bar{\varepsilon}$

$$
\tilde{\mathbf{u}}=\mathbf{u}-\bar{\varepsilon} \mathbf{x} \text { on } \partial \Omega \text {. }
$$

The cell boundary $\partial \Omega$ is decomposed into two parts: $\partial \Omega^{+}$and $\partial \Omega^{-}$with $\partial \Omega^{+} \cup \partial \Omega^{-}=$ $\partial \Omega, \partial \Omega^{+} \cap \partial \Omega^{-}=\varnothing$, and the associated outward normals $\mathbf{n}^{+}=-\mathbf{n}^{-}$at corresponding points $\mathbf{x}^{+} \in \partial \Omega^{+}$and $\mathbf{x}^{-} \in \partial \Omega^{-}$, respectively. The periodic boundary condition represents the periodicity of the displacement fluctuation field and anti-periodicity of the traction field on the cell boundary

$$
\begin{aligned}
& \tilde{\mathbf{u}}\left(\mathbf{x}^{+}\right)=\tilde{\mathbf{u}}\left(\mathbf{x}^{-}\right) \text {on } \partial \Omega^{+}, \\
& \mathbf{t}\left(\mathbf{x}^{+}\right)=-\mathbf{t}\left(\mathbf{x}^{-}\right) \text {on } \partial \Omega^{-},
\end{aligned}
$$

where the traction field $\mathbf{t}$ on the boundary $\partial \Omega$ is expressed by

$$
\mathbf{t}=\sigma \mathbf{n} \text {. }
$$

The conform mesh is used to capture all inhomogeneity interfaces. It's generated in ANSYS so that the nodes in boundary are symmetric about their vertical and horizontal center axes. Using the FEM procedure, we obtain the discrete system of linear ordinary equations

$$
\mathbf{K d}=\mathbf{F},
$$

where $\mathbf{d}$ is nodal unknown vector, and $\mathbf{K}$ and $\mathbf{F}$ are the global stiffness matrix and external force vector, respectively. In particular, the matrix $\mathbf{K}$ and vector $\mathbf{F}$ are defined by

$$
\mathbf{K}=\int_{\Omega} \mathbf{B}^{T} \mathbf{C B} \mathrm{d} \Omega, \quad \mathbf{F}=\int_{\Gamma_{t}} \mathbf{N}^{T} \overline{\mathbf{t}} \mathrm{d} \Gamma+\int_{\Omega} \mathbf{N}^{T} \mathbf{b} \mathrm{d} \Omega,
$$

where $\mathbf{B}$ and $\mathbf{N}$ are the matrices of shape function derivatives and shape functions, and $\mathbf{C}$ is the matrix corresponding to the elasticity tensor $\mathbb{C}$. The periodic boundary conditions (21) are finally introduced by mean of Lagrange multipliers.

As the first numerical example, we consider two-component 2D square-periodic cell involving 4 elliptic inclusions making with the boundary an angle $\theta=45^{\circ}$ as illustrated in Fig. 1a. The equivalent configuration is graphically presented in Fig. 1b. Aspect ratio of the ellipses takes the same value $r=2$. The calculations are carried out for 4 cases corresponding the different material properties $K_{M}, K_{I}, \mu_{M}, \mu_{I}$ and equivalent-inclusions properties $\bar{K}_{I}, \bar{\mu}_{I}$ according to (16)-(17):

a) $K_{M}=10, K_{I}=1, \mu_{M}=2, \mu_{I}=0.4, \bar{K}_{I}=0.8212, \bar{\mu}_{I}=0.3629$, 
b) $K_{M}=1, K_{I}=10, \mu_{M}=2, \mu_{I}=0.4, \bar{K}_{I}=6.9577, \bar{\mu}_{I}=0.4289$,

c) $K_{M}=10, K_{I}=1, \mu_{M}=0.4, \mu_{I}=2, \bar{K}_{I}=1.0463, \bar{\mu}_{I}=1.9283$,

d) $K_{M}=1, K_{I}=10, \mu_{M}=0.4, \mu_{I}=2, \bar{K}_{I}=12.8297, \bar{\mu}_{I}=2.1166$.

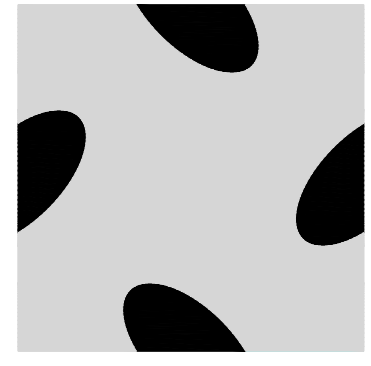

a)

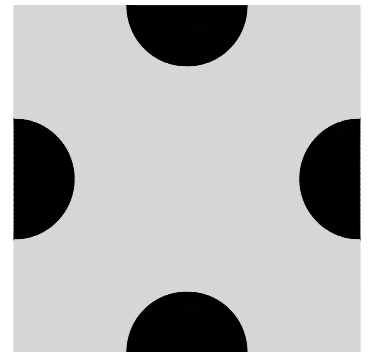

b)

Fig. 1. The periodic square cell with four semi elliptic inclusions (a) and the equivalent configuration (b)
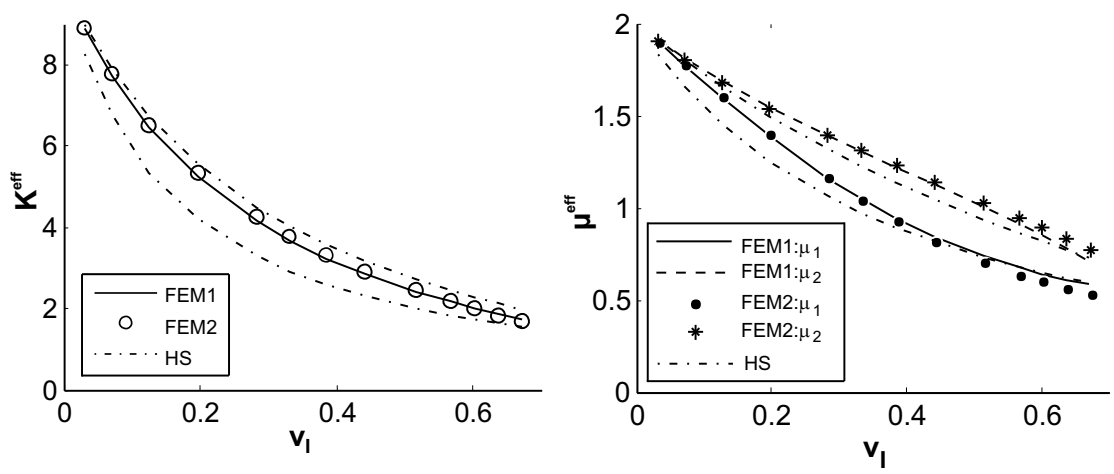

Fig. 2. Effective elastic moduli of the square periodic composite with material properties $K_{M}=10, K_{I}=1, \mu_{M}=2, \mu_{I}=0.4, \bar{K}_{I}=0.8212, \bar{\mu}_{I}=0.3629$

The effective elastic moduli are presented in Figs. 2-5 where $\mu_{1}^{\text {eff }}$ and $\mu_{2}^{\text {eff }}$ refer to the shear moduli in edge direction and diagonal direction respectively (since the composite is not fully isotropic); FEM1 and FEM2 corresponding to the results for original and equivalent configuration. The moduli $K^{\text {eff }}, \mu_{1}^{\text {eff }}$ and $\mu_{2}^{\text {eff }}$ are obtained by solving 3 problems associated with the macroscopic strains $\bar{\varepsilon}$ as follow

$$
\bar{\varepsilon}_{1}=\left[\begin{array}{ll}
1 & 0 \\
0 & 0
\end{array}\right], \bar{\varepsilon}_{2}=\left[\begin{array}{ll}
0 & \frac{1}{2} \\
\frac{1}{2} & 0
\end{array}\right], \quad \bar{\varepsilon}_{3}=\left[\begin{array}{cc}
1 & 0 \\
0 & -1
\end{array}\right] .
$$

The approximations become less accurate at higher proportions of the included phase - that is inevitable as the equivalent inclusion approach, based on equalizing of effective moduli of the dilute solution results for specified form of inclusions, can not 

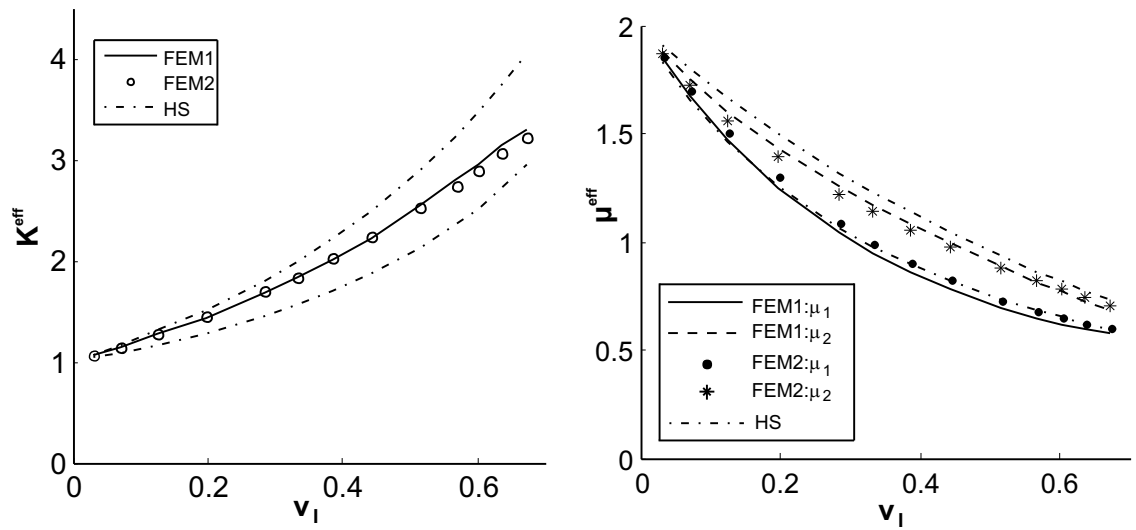

Fig. 3. Effective elastic moduli of the square periodic composite with material properties $K_{M}=1, K_{I}=10, \mu_{M}=2, \mu_{I}=0.4, \bar{K}_{I}=6.9577, \bar{\mu}_{I}=0.4289$
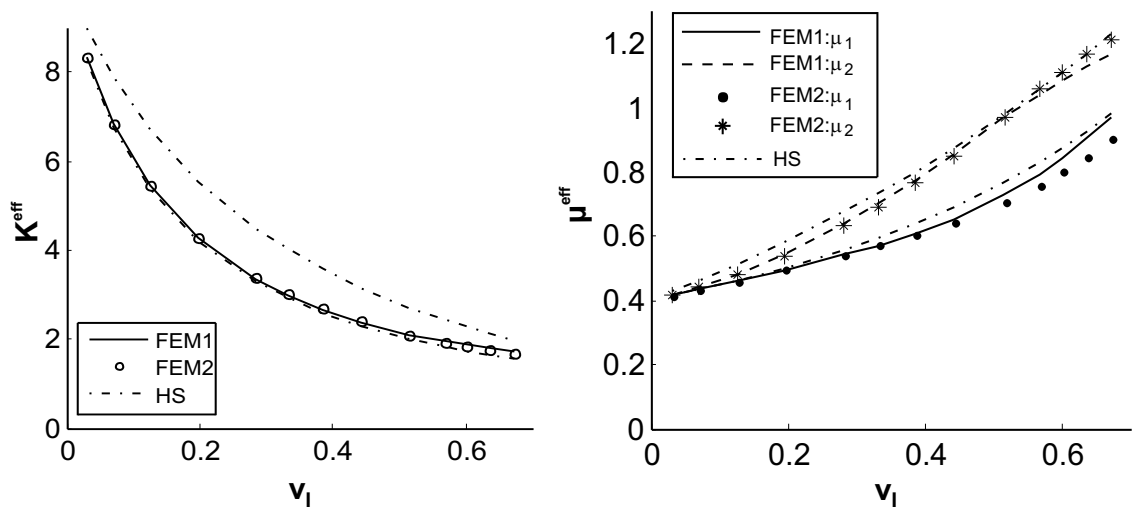

Fig. 4. Effective elastic moduli of the square periodic composite with material properties $K_{M}=10, K_{I}=1, \mu_{M}=0.4, \mu_{I}=2, \bar{K}_{I}=1.0463, \bar{\mu}_{I}=1.9283$
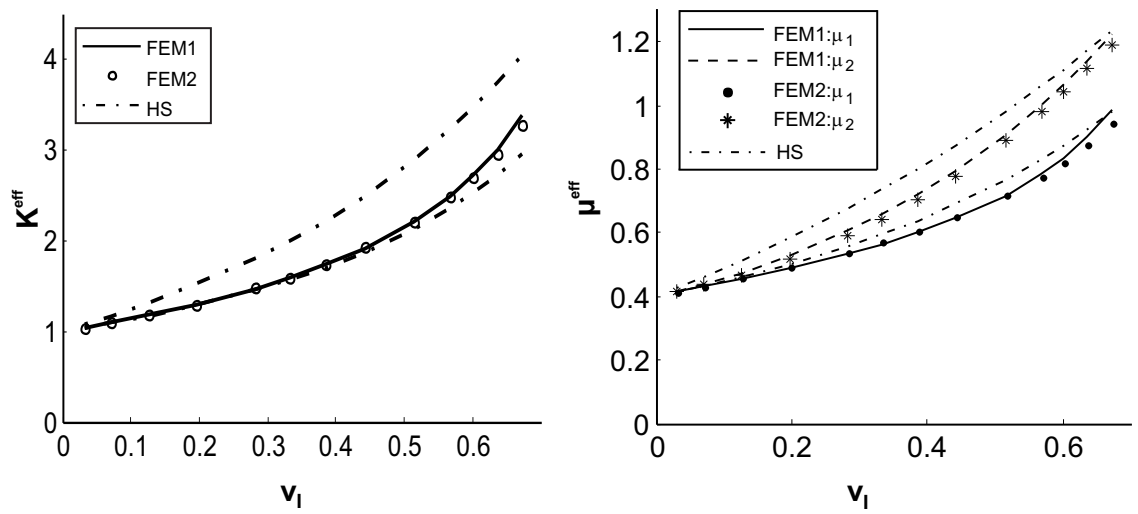

Fig. 5. Effective elastic moduli of the square periodic composite with material properties $K_{M}=1, K_{I}=10, \mu_{M}=0.4, \mu_{I}=2, \bar{K}_{I}=12.8297, \bar{\mu}_{I}=2.1166$ 
take into account accurately the detailed interactions between the inclusions in close distances. However, the approximation appears good regarding its simplicity and generality. The numerical results for the shear moduli appear not to obey HS bounds because the configurations are not fully elastically isotropic.

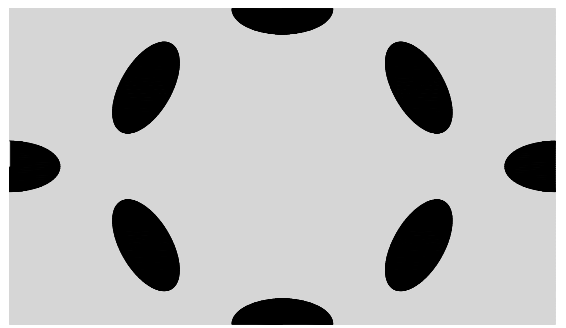

a)

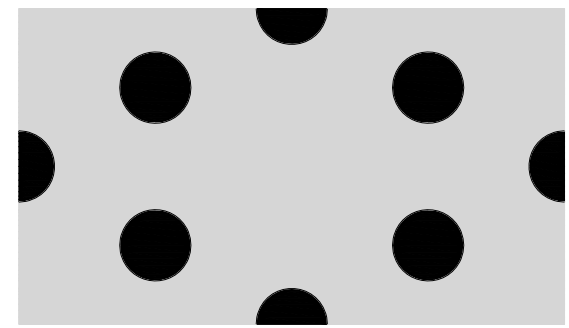

b)

Fig. 6. The hexagonal-periodic cell with elliptic inclusions (a) and the equivalent configuration (b)
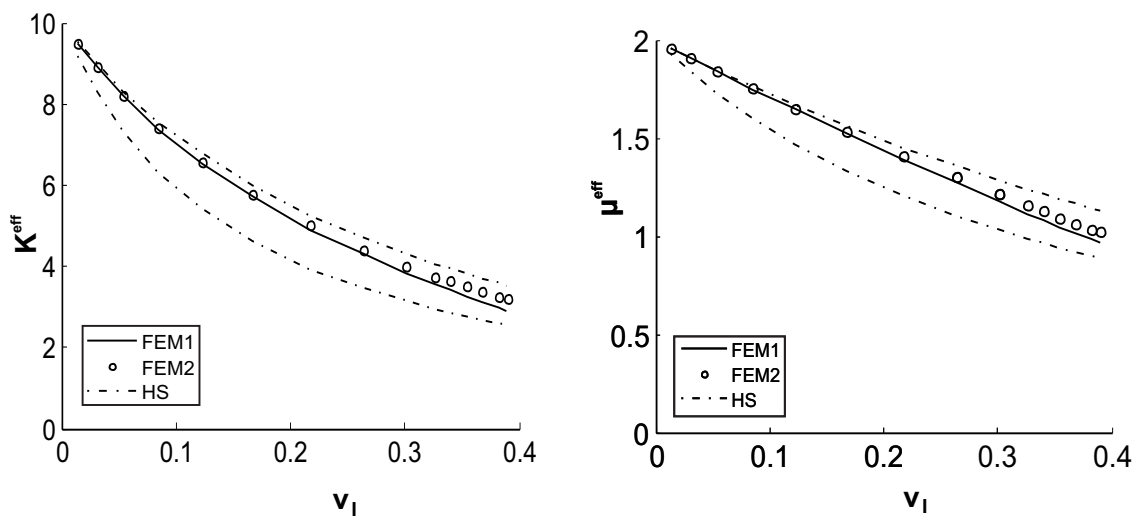

Fig. 7. Effective elastic moduli of the hexagonal periodic composite with material properties

$$
K_{M}=10, K_{I}=1, \mu_{M}=2, \mu_{I}=0.4, \bar{K}_{I}=0.8212, \bar{\mu}_{I}=0.3629
$$

In the second example, we consider an elastically-isotropic composite of hexagonal symmetry, a periodic cell of which is illustrated in the Fig. 6a. The equivalent configuration is graphically presented in Fig. 6b. Aspect ratio of the ellipses takes the same value $r=2$. The elastic moduli of the two components are taken as the same as those of the previous example. Numerical results for the effective elastic moduli of both two configurations, presented in Figs. 7-10, are obtained by solving 2 problems with the macroscopic strains $\bar{\varepsilon}_{1}$ and $\bar{\varepsilon}_{2}$. The effective elastic moduli of the original medium (FEM1) and one of the equivalent medium (FEM2) always lie between the bounds of Hashin and Shtrikman (HS) as expected. Similar to the previous example, the inaccuracies of the approximations increase with the volume proportion of the inclusions. In Fig. 7, with harder matrix $K_{M}>K_{I}$ and $\mu_{M}>\mu_{I}$, the numerical effective moduli are close to the HS upper bounds 

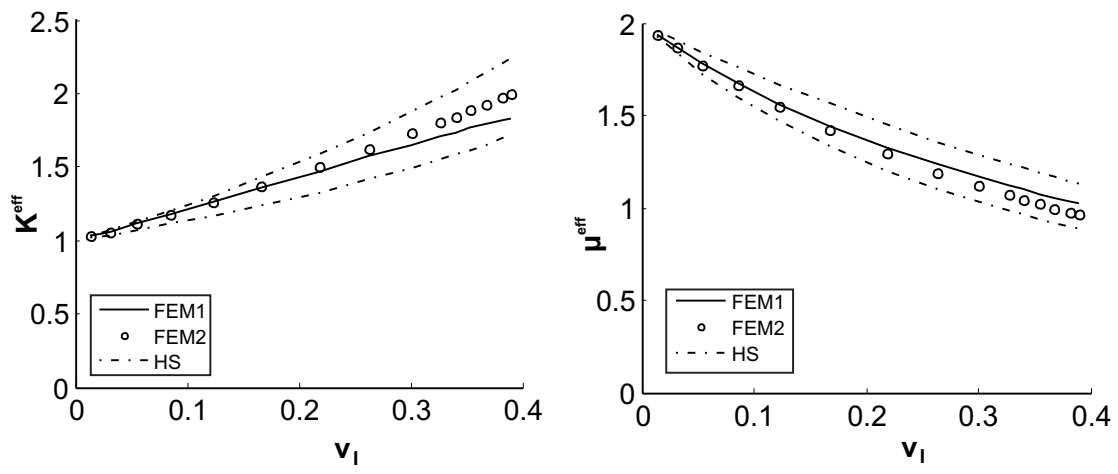

Fig. 8. Effective elastic moduli of the hexagonal periodic composite with material properties $K_{M}=1, K_{I}=10, \mu_{M}=2, \mu_{I}=0.4, \bar{K}_{I}=6.9577, \bar{\mu}_{I}=0.4289$
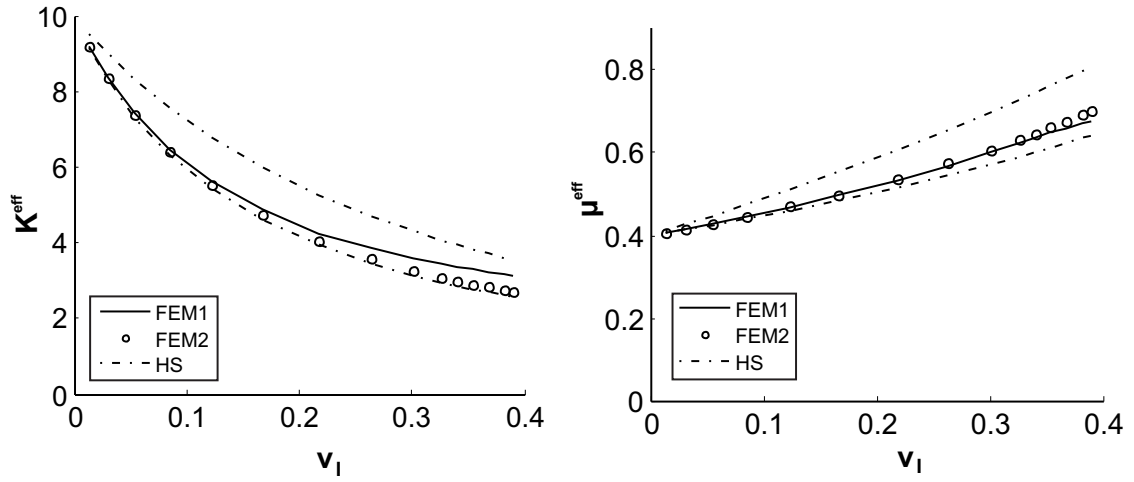

Fig. 9. Effective elastic moduli of the hexagonal periodic composite with material properties $K_{M}=10, K_{I}=1, \mu_{M}=0.4, \mu_{I}=2, \bar{K}_{I}=1.0463, \bar{\mu}_{I}=1.9283$
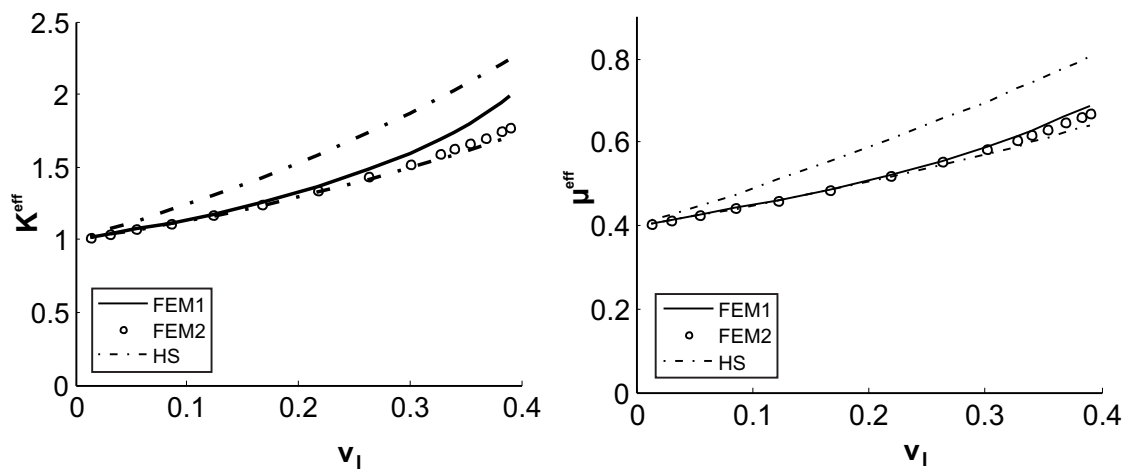

Fig. 10. Effective elastic moduli of the hexagonal periodic composite with material properties $K_{M}=1, K_{I}=10, \mu_{M}=0.4, \mu_{I}=2, \bar{K}_{I}=12.8297, \bar{\mu}_{I}=2.1166$ 
at small volume proportions of the inclusions as expected. However at high volume proportions of the included phase the results tend to take the side of HS lower bound that because the inclusions have the tendency to connect with each other to make a new pseudo-matrix surrounding and separating the islands of the original matrix phase (look back at Fig. 6). In Fig. 8 the tendency is mixed and unpredictable because $K_{M}<K_{I}$ but $\mu_{M}>\mu_{I}$. The influence of $r$ to the approaches accuracy shall be the subject of our future studies.

\section{CONCLUSION}

We have proposed a novel approach which replaces the original composite with elliptic inclusions by the equivalent-composite with isotropic circular-inclusions for which many available simple approximations can be applied to estimate the effective elastic moduli. The proposed approach is based on equalizing of effective moduli of the dilute solution results for the elliptic inclusions and those of the standard circular-inclusions. The numerical examples have demonstrated the robustness, efficiency and accuracy of the approach. 3D composite and inclusion of other forms shall be the subject of our future studies.

\section{ACKNOWLEDGEMENT}

This work is funded by Vietnam's National Foundation for Science and Technology Development, Project N. 107.02-2013.20.

\section{REFERENCES}

[1] Z. Hashin and S. Shtrikman. A variational approach to the theory of the effective magnetic permeability of multiphase materials. Journal of Applied Physics, 33, (10), (1962), pp. 31253131.

[2] M. N. Miller. Bounds for effective electrical, thermal, and magnetic properties of heterogeneous materials. Journal of Mathematical Physics, 10, (11), (1969), pp. 1988-2004.

[3] N. Phan-Thien and G. W. Milton. New bounds on the effective thermal conductivity of Nphase materials. Proceedings of the Royal Society of London. A. Mathematical and Physical Sciences, 380, (1779), (1982), pp. 333-348.

[4] C. K. Le and D. C. Pham. On bounding the effective conductivity of isotropic composite materials. Zeitschrift für Angewandte Mathematik und Physik ZAMP, 42, (4), (1991), pp. 614622.

[5] D. C. Pham. Bounds on the effective conductivity of statistically isotropic multicomponent materials and random cell polycrystals. Journal of the Mechanics and Physics of Solids, 59, (3), (2011), pp. 497-510.

[6] D. C. Pham, L. D. Vu, and V. L. Nguyen. Bounds on the ranges of the conductive and elastic properties of randomly inhomogeneous materials. Philosophical Magazine, 93, (18), (2013), pp. 2229-2249.

[7] C. F. Dunant, B. Bary, A. B. Giorla, C. Péniguel, J. Sanahuja, C. Toulemonde, A.-B. Tran, F. Willot, and J. Yvonnet. A critical comparison of several numerical methods for computing effective properties of highly heterogeneous materials. Advances in Engineering Software, 58, (2013), pp. 1-12. 
[8] B. Bary, M. B. Haha, E. Adam, and P. Montarnal. Numerical and analytical effective elastic properties of degraded cement pastes. Cement and Concrete Research, 39, (10), (2009), pp. 902912.

[9] A.-B. Tran, J. Yvonnet, Q.-C. He, C. Toulemonde, and J. Sanahuja. A multiple level set approach to prevent numerical artefacts in complex microstructures with nearby inclusions within XFEM. International Journal for Numerical Methods in Engineering, 85, (11), (2011), pp. 1436-1459.

[10] H. Moulinec and P. Suquet. A fast numerical method for computing the linear and nonlinear mechanical properties of composites. Comptes Rendus de l'Académie des Sciences. Série II, Mécanique, Physique, Chimie, Astronomie, 318, (11), (1994), pp. 1417-1423.

[11] J. C. Michel, H. Moulinec, and P. Suquet. A computational scheme for linear and non-linear composites with arbitrary phase contrast. International Journal for Numerical Methods in Engineering, 52, (1-2), (2001), pp. 139-160.

[12] J. Sanahuja and C. Toulemonde. Numerical homogenization of concrete microstructures without explicit meshes. Cement and Concrete Research, 41, (12), (2011), pp. 1320-1329.

[13] A. N. Norris, A. J. Callegari, and P. Sheng. A generalized differential effective medium theory. Journal of the Mechanics and Physics of Solids, 33, (6), (1985), pp. 525-543.

[14] M. Milgrom and S. Shtrikman. A layered-shell model of isotropic composites and exact expressions for the effective properties. Journal of Applied Physics, 66, (8), (1989), pp. 3429-3436.

[15] D. C. Pham. Estimations for the overall properties of some locally-ordered composites. Acta Mechanica, 121, (1-4), (1997), pp. 177-190.

[16] N. Phan-Thien and D. C. Pham. Differential multiphase models for polydispersed spheroidal inclusions: thermal conductivity and effective viscosity. International Journal of Engineering Science, 38, (1), (2000), pp. 73-88.

[17] D. C. Pham and S. Torquato. Strong-contrast expansions and approximations for the effective conductivity of isotropic multiphase composites. Journal of Applied Physics, 94, (10), (2003), pp. 6591-6602.

[18] D. C. Pham. Weighted effective medium approximations for conductivity of random composites. International Journal of Heat and Mass Transfer, 51, (13), (2008), pp. 3355-3361.

[19] H. Le Quang, G. Bonnet, and D. C. Pham. Bounds and correlation approximation for the effective conductivity of heterogeneous plates. Physical Review E, 84, (6), (2011). DOI: 10.1103/PhysRevE.84.061153.

[20] D. C. Pham, A. B. Tran, and Q. H. Do. On the effective medium approximations for the properties of isotropic multicomponent matrix-based composites. International Journal of Engineering Science, 68, (2013), pp. 75-85.

[21] D. C. Pham. Essential solid mechanics. Institute of Mechanics, Hanoi, (2013).

[22] T. Mura. Micromechanics of defects in solids. Kluwer, Dordecht, (1987).

[23] R. M. Christensen. Mechanics of composite materials. Wiley, New York, (1979). 